\title{
OPEN Detection and identification of drug traces in latent fingermarks using Raman spectroscopy
}

\begin{abstract}
Mohamed O. Amin ${ }^{1}$, Entesar Al-Hetlani ${ }^{1 \bowtie}$ \& Igor K. Lednev ${ }^{2 \bowtie}$
Recent advancements in analytical techniques have greatly contributed to the analysis of latent fingermarks' (LFMs) "touch chemistry" and identification of materials that a suspect might have come into contact with. This type of information about the FM donor is valuable for criminal investigations because it narrows the pool of suspects. It is estimated that at least $\mathbf{3 0}$ million people around the world take over-the-counter and prescription nonsteroidal anti-inflammatory drugs (NSAIDs) for pain relief, headaches and arthritis every day. The daily use of such drugs can lead to an increased risk of their abuse. In the present study, Raman spectroscopy combined with multivariate statistical analysis was used for the detection and identification of drug traces in LFMs when NSAID tablets of aspirin, ibuprofen, diclofenac, ketoprofen and naproxen have been touched. Partial least squares discriminant analysis of Raman spectra showed an excellent separation between natural FMs and all NSAIDcontaminated FMs. The developed classification model was externally validated using FMs deposited by a new donor and showed $100 \%$ accuracy on a FM level. This proof-of-concept study demonstrated the great potential of Raman spectroscopy in the chemical analysis of LFMs and the detection and identification of drug traces in particular.
\end{abstract}

Fingerprints are among the greatest discoveries of forensic sciences and have been employed for decades as the primary biometric means of human individualization. Establishment of a clear connection between a questioned fingermark (FM) and an individual entails a minute comparison of the morphological pattern of the FM with a reference material (e.g., a fingerprint from a suspect $)^{1}$. However, this forensic application of FM is based on the comparison with a known FM either collected from a suspect or found in the database. The biochemical composition of a FM contains numerous information about the donor, including age ${ }^{2}, \operatorname{sex}^{3}$, health status ${ }^{4}$ and other characteristics that may have been previously overlooked. Such characteristics can be identified by the presence of thousands of compounds in FM residue originating from both endogenous and exogenous components. Endogenous substances result from the natural secretion of the human body, such as lipids, waxes, amino acids, and proteins, while substances that come in contact with fingertips prior to deposition (e.g., drugs, cosmetics, explosives) are often referred to as exogenous substances. In addition, semi-exogenous substances can also be found in FMs; these substances result from compounds that are ingested and then excreted in their metabolized and unmetabolized forms through sweat (e.g. drugs, food and drink components) ${ }^{5}$.

These exogenous substances reflect information about the individual's lifestyle and therefore have been the focus of several recent studies ${ }^{6}$. Thus, chemical analysis of the contaminants in FMs has served as a mainstay in forensic investigations, producing prosecutorial evidentiary data ${ }^{5}$. For this purpose, several analytical techniques have been employed for the chemical analysis of FMs, such as gas chromatography-mass spectrometry $(\mathrm{GC}-\mathrm{MS})^{7}$, ultra-performance liquid chromatography-mass spectrometry (UPLC-MS) ${ }^{8}$, laser desorption/ ionization-mass spectrometry (LDI-MS) ${ }^{9}$ and others.

Owing to their inherent nondestructive nature and the need for little to no sample pretreatment, vibrational spectroscopic techniques, including Raman spectroscopy, Fourier transform infrared (FTIR) spectroscopy and attenuated total reflection Fourier transform infrared (ATR-FTIR) spectroscopy, have great potential in forensic applications ${ }^{10-12}$. Vibrational spectroscopy has garnered a considerable amount of attention in the field of FM analysis ${ }^{13}$. A comprehensive review by Ewing and Kazarian discussed the use of vibrational spectroscopic imaging to obtain the chemical composition of FMs and exogenous materials ${ }^{14}$. In particular, ATR-FT-IR spectroscopic imaging has been utilized for the analysis of latent FM and its changes under controlled humidity and temperature ${ }^{15,16}$. Furthermore, Kazarian and coworkers have investigated the use of common adhesive tape

${ }^{1}$ Department of Chemistry, Kuwait University, Faculty of Science, P.O. Box 5969, 13060 Safat, Kuwait. ${ }^{2}$ Department of Chemistry, University at Albany, SUNY, 1400 Washington Avenue, Albany, NY 12222, USA. "घemail: entesar.alhetlani@ku.edu.kw; ilednev@albany.edu 
and polydimethylsiloxane (PDMS) film along with ATR spectroscopy to detect drugs of abuse ${ }^{17}$. Commercial gel lifters and ATR-FTIR spectroscopy have been used to generate chemical imaging from a different depth of $\mathrm{FMs}^{18}$. In addition, FM contaminated with cosmetics on porous and nonporous surfaces have been analyzed ${ }^{19}$.

Raman spectroscopy, through its technological advancement, has enabled efficient detection of several forensically relevant contaminants and household products in FM. For instance, significant strides have been made in the detection of explosive residues in FMs, which is of importance in preventing terroristic attacks on civilians ${ }^{20,21}$. In addition, drug-contaminated FMs are valuable evidence that can be used to reconstruct various types of crime scenes, such as suicide, drug abuse, drug manufacturing and others. In this regard, Raman spectroscopy has been applied in two different studies that detected drugs of abuse ${ }^{22}$, including codeine, cocaine, amphetamine, barbital and nitrazepam and adulterants, as well as caffeine, aspirin, paracetamol, starch and talc powders ${ }^{23}$ in FM impressions. Furthermore, FMs contaminated with the drugs of abuse including ecstasy, cocaine, ketamine and amphetamine have been lifted with adhesive tape and analyzed using Raman spectroscopy ${ }^{24}$. In another study by West and coworkers ${ }^{25}$, Raman spectroscopy was employed for the analysis of FMs contaminated with over-the-counter (OTC) analgesic substances. These FMs had been treated with powder and lifted with adhesive tape. The findings indicated that the application of powders did not hinder the identification of contaminants in FM impressions. Additionally, analysis of drug powder and additive doping in FMs was investigated using tape lifting and Raman microscopy; the obtained spectra were deconvoluted using the multivariate technique Bandtarget entropy minimization (BTEM) ${ }^{26}$. These combined methods enabled the identification of the test substances using their characteristic Raman signatures. Based on the above literature, limited studies have focused on the analysis of drug-contaminated FMs using Raman spectroscopy despite its importance in forensic cases.

This study expands the potential use of Raman spectroscopy for detecting drugs in contaminated FMs obtained by gentle touching nonsteroidal anti-inflammatory drug (NSAIDs) pharmaceutical tablets purchased from a local drugstore. Two important aspects of this approach for preparing mock contaminated FMs are considered. (1) NSAIDs pharmaceutical tablets contain active ingredient(s) as well as other excipients that makes the mock samples more realistic. (2) Touching a tablet is practically important and further expands the variety of drug-contaminated FMs, which can be analyzed by Raman spectroscopy. Five commonly used NSAID tablets were selected for this study and subjected to Raman analysis. These drugs share many of the same functional groups, and subtle differences can be seen in their Raman spectra when they are present in FMs. Therefore, the differentiation of FM samples was evaluated using multivariate data analysis. Initially, principal component analysis (PCA) was carried out on the preprocessed spectra for the removal of multivariate outliers, defined as spectra with high Hoteling's $\mathrm{T}^{2}$ and $\mathrm{Q}$ residuals values in all the datasets, and they were removed before any further statistical analysis was performed. Further advanced statistical analysis was applied, including a genetic algorithm (GA), to select the most informative features in the spectra in the training process of partial least squares-discriminate analysis (PLS-DA) to distinguish between FM classes. Both internal and external validations were applied to evaluate the performance of the model. The obtained results clearly demonstrate that Raman spectroscopy is a powerful analytical method that can detect traces of drugs in FMs and define the type of drug with which the FM made contact.

\section{Experimental}

Sample preparation. Initially, hands were washed with water and soap and then thoroughly dried. The donor was requested to rub his index finger on the forehead, nose and chin five times to eventually produce a sebum-rich mark and to stimulate natural grooming behavior, after which the finger was placed directly on a microscope slide covered with aluminum adhesive tape (Nashua tape, Home Depot). The NSAID-contaminated FMs were prepared as follows: the donor was requested to rub his index on the forehead, nose and chin five times and then touch a pharmaceutical tablet purchased from a local drugstore containing one of these active ingredients: aspirin (500 mg), ibuprofen (400 mg), diclofenac (50 mg), ketoprofen (100 mg) or naproxen (500 $\mathrm{mg})$ for $10 \mathrm{~s}$. The contaminated FMs were applied to a microscope slide covered with aluminum tape for Raman spectroscopic analysis. All FMs were analyzed within $30 \mathrm{~min}$ post collection. All procedures were approved by the Health Sciences Centre Ethical Committee of Kuwait University and in accordance with the ethical standards of the institutional and/or national research committee and with the 1964 Helsinki Declaration and its later amendments or comparable ethical standards. An informed consent was obtained from all subjects and/or their legal guardian(s).

Instrumentation and spectra collection. Spectra of the FMs were acquired utilizing a Renishaw inVia confocal Raman microscope with a $1200 \mathrm{~L} / \mathrm{mm}$ grating and a CCD camera. The analyses were carried out using a $785 \mathrm{~nm}$ diode laser for excitation, and the spectra were collected in the range of 400 to $1800 \mathrm{~cm}^{-1}$, employing $50 \%$ laser power and $20 \mathrm{~s}$ exposure time. A longitudinal spot was attained at a high optical magnification of xL50, and WiRe 4.4 software was used to control the microscope. The instrument was calibrated with a silicon standard prior to analysis, and a vibrational band corresponding to a silicon wafer was used for this purpose. An automatic mapping stage was used for the analysis, and a total of 52-56 spectra were obtained from different spots to account for sample heterogeneity.

Data analysis. Dataset preparation and statistical analysis were carried out using PLS Toolbox 8.9.1 (Eigenvector Research, Inc., Wenatchee, WA) operating in MATLAB R2020b (MathWorks, Inc., Natick, MA) ${ }^{27}$. All the spectra were baseline-corrected by automatic weighted squares, normalized and mean centered. A PCA model was constructed to identify multivariate outliers, which were removed before any further statistical analyses. Genetic algorithm (GA) was used to determine the main spectral features to be included in the training process of PLS-DA. A population size of 64 and generation number of 100 were used in the GA spectral selection. Dou- 
ble crossover was set for the breeding crossover rule, and the mutation rate was 0.005 . A supervised learning technique, PLS-DA, was applied to distinguish between different FM classes and identify the drug(s) present in the questioned FMs.

Validation tests. In this study, the internal validation of the PLS-DA model was carried out using the Venetian blinds method of cross validation (CV) with ten splits. In theory, the internal CV allows for testing how well the available dataset supports the classification hypothesis. A true validation of a classification model requires its testing on an external data, which is not used for building the model and has a size of at least $30-40 \%$ of the training dataset. For these reason, we conducted both an internal CV and external validation. The Venetian blinds $\mathrm{CV}$ involved a sequence of steps, in which a subset of ten spectra was removed from the training dataset and a sub-model was constructed using the remaining spectra. As a result, each sub-model was tested with spectra, which have not been used to build the model. The iterations were continued until each spectrum in the training dataset was removed once.

To further increase the reliability of the proposed method, an external validation using an independent dataset was employed. In this study, natural and contaminated FMs were obtained from a second donor following the same procedure mentioned above. The spectra were baseline-corrected by automatic weighted squares, normalized and mean centered and introduced to the PLS-DA model for prediction purposes. This validation demonstrated that the classification model built based on contaminated FMs obtained from one donor can be reliably applied for determining the NSAID drugs in contaminated FMs obtained from another donor.

\section{Results and discussion}

Spectral analysis of NSAIDs contaminated FM. FM residue is a complex mixture composed of numerous compounds originating from different sources and includes organic constituents (e.g., protein, lipids, vitamins and amino acids), ions $\left(\mathrm{Cl}^{-}, \mathrm{K}^{+}, \mathrm{Na}^{+}, \mathrm{Mg}^{2+}\right)$ and trace metals $(\mathrm{Zn}, \mathrm{Cu}, \mathrm{Fe})$ resulting from the natural secretions of the $\operatorname{skin}^{5}$. While several factors can influence the chemical composition of FMs such as donor, environmental conditions, type of substrate, and substances that may have come in contact with the fingertips, such as drugs, cosmetics and explosives. Specifically, drug-contaminated FMs can be discovered in a variety of crime scenes, including suicide cases, drug abuse, drug handling or street drug diluents that pertain to several different forensic scenarios. Due to the large number of drugs that can be employed for these purposes, we selected NSAIDs tablets for our proof-of-concept study because they are common drugs that can be found in any household and can be purchased OTC without a prescription at a low cost. Additionally, despite NSAIDs are supposed to be safe drugs, they may lead to severe toxic effects in cases of acute overdosage and chronic abuse. NSAIDs misuse has been also reported in horse doping, therefore, they may be encountered in clinical, forensic toxicological analyses and in horse doping control $^{28}$.

Aluminum foil with an adhesive layer has been used to cover a glass slide and deposit the FMs Aluminum foil is an ideal substrate for Raman spectroscopy because it is readily available, inexpensive and most importantly it does not result in any significant interfering signal ${ }^{29,30}$. A minimal (gentle) pressure was applied on the tablet by the fingers, which was just sufficient to hold the tablet, to mimic a real-life situation. A holding time of about $10 \mathrm{~s}$ was used to make mock FMs samples. Although we did not investigate these two factors (pressure and time) quantitatively, no inconsistency or irreproducibility were noticed when the Raman spectra were analyzed. Most importantly, we were able to develop accurate classification model for drug differentiation.

Initially, FMs contaminated with aspirin were obtained from all five fingers of the left hand of one donor. Average Raman spectra collected from each of those FMs (Figure S1) are similar to each other indicating that the intra-donor variability has minimum effect. Consequently, the analysis of FMs contaminated with various drugs was conducted in this study using the index finger only. In this study, Raman spectroscopy was employed to detect traces of NSAID tablets in FMs and distinguish between natural FM components and FMs after contact with different NSAID tablets. Five pharmaceutical tablets containing aspirin, ibuprofen, diclofenac, ketoprofen and naproxen as active ingredients were utilized to produce NSAID-contaminated FMs. 54-56 Raman spectra were obtained from each FM sample using automatic mapping to account for the inherent heterogeneity of the sample. The Raman spectra of the natural and contaminated FMs showed variation in peak position and intensity, reflecting the differences in the chemical compositions of the FM samples, as illustrated in Fig. 1. The peaks corresponding to the eccrine and sebaceous components of the natural FMs, including $\mathrm{C}=\mathrm{O}$ stretching from the secondary amide, $\mathrm{CH}_{2}$ deformation and twisting from the aliphatic chain, and $\mathrm{C}=\mathrm{CH}$ deformation from squalene and unsaturated fatty acids, were clearly observed in all the spectra ${ }^{31}$. Additional Raman bands were observed in the spectra of the NSAID-contaminated FMs resulting from the contribution of the active tablet ingredients and other excipients present in the product. The most prominent band was attributed to ring stretching and was observed at $1598 \mathrm{~cm}^{-1}$ and $1629 \mathrm{~cm}^{-1}$ for the ketoprofen- and naproxen-containing tablets, respectively, while it was observed at $1606 \mathrm{~cm}^{-1}$ for the aspirin-, ibuprofen- and diclofenac-containing tablets. Table 1 shows the tentative assignment of the Raman bands of the natural FM and NSAID-contaminated FMs based on previous literature. Although Raman spectroscopy was able to detect traces of NSAIDs in the contaminated FM residues, we exploited chemometrics herein to generate a statistical model to classify and differentiate between the respective FM samples. This is the first step in identifying the components of natural FM and FMs generated after certain drug tablets were touched.

Statistical analysis of Raman spectral data. Statistical modeling was employed to study the variation in the Raman spectra of the natural and contaminated FMs, which account for the different functional groups present in the sample, as shown in Table 1. Initially, an unsupervised learning technique, PCA model, was applied to explore the dataset and to reduce the dimensionality of the multivariate data by generating several 


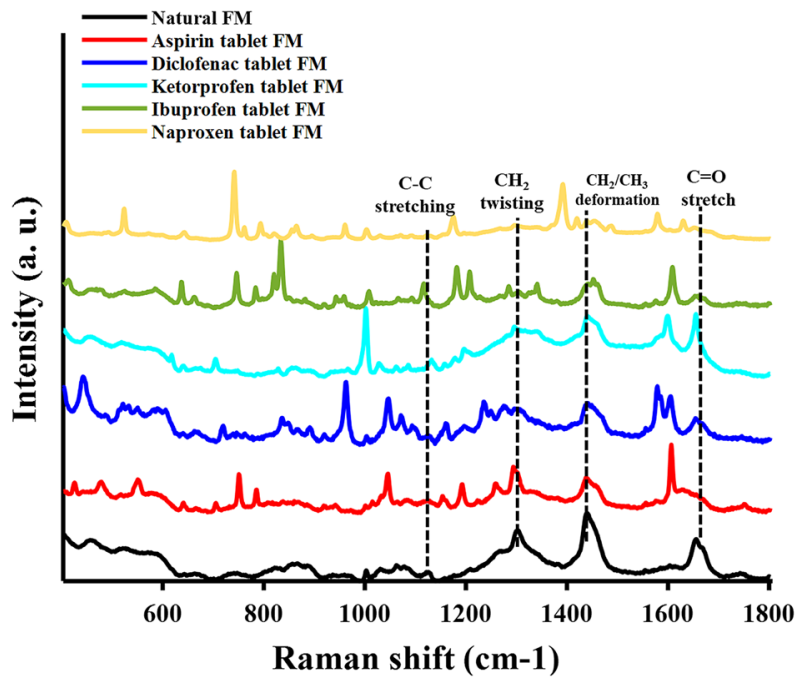

Figure 1. The average Raman spectra of natural fingermark and fingermarks contaminated with aspirin, diclofenac, ketoprofen, ibuprofen or naproxen tablets. The experimental spectra were preprocessed by baseline correction and normalization.

uncorrelated variables that successfully capture the maximum variance in the dataset. Multivariate outliers were removed through PCA, defined as spectra with high Hoteling's $\mathrm{T}^{2}$ and Q residuals resulting in a total of 225 spectra: 31 spectra of natural FM, 45 spectra of aspirin tablet FM, 43 spectra of diclofenac tablet FM, 28 spectra of ibuprofen tablet FM, 47 spectra of ketoprofen tablet FM and 31 spectra of naproxen tablet FM. The first five PCs described $84.5 \%$ of the total variance. For representation purposes, the PCA score plot of three PCs (PC 1, PC 2 and PC 5) for different FM samples is depicted in Fig. 2. This figure suggests that the three PCs were able to separate the dataset into six different groups corresponding to natural FM and aspirin-, diclofenac-, ibuprofen-, ketoprofen- and naproxen-contaminated FMs. However, the spectra of some of the drug-contaminated FM samples overlapped with those of the natural FM in the PCs projection. This result can be attributed to the contribution of the FM components in all the datasets. As PCA is an unsupervised statistical discrimination tool, such a contribution is expected to be prominent in the statistical model. Therefore, to provide sufficient separation between FM samples, a supervised learning technique, PLS-DA, was employed on the dataset.

Differentiating between natural fingermarks and NSAID tablet-contaminated fingermarks using partial least squares-discriminant analysis (PLS-DA). PLS-DA is a versatile algorithm that can be employed for prediction and description as well as discrimination of variable selections. In theory, PLSDA is a linear classification method that combines dimensionality reduction and discrimination power of the classification technique, thereby offering a high differentiation power that can be used for class prediction within the datasets ${ }^{38}$. Therefore, PLS-DA has shown great potential in modeling multivariate datasets for different purposes, including food analysis ${ }^{39}$, disease diagnosis ${ }^{40}$, and, more specifically, analysis of forensic evidence ${ }^{41}$. For instance, Lednev and coworkers pioneered the identification of all main bodily fluids for forensic purposes using PLS-DA model ${ }^{42}$. High levels of discrimination between human and animal blood, menstrual and peripheral blood and phenotype profiling based on bloodstain analysis have been achieved using PLS-DA model combined with ATR FTIR spectroscopy ${ }^{43-45}$. In the present study, we used this method to detect and identify five different drugs in contaminated FMs. We accomplished these goals by differentiating six classes of FMs, including natural FM that were free of any drugs and FMs contaminated with five different drugs. Specifically, after the spectra were baseline-corrected by automatic weighted squares, normalized by total area and mean centered, a PLS-DA model was built using five latent variables (LVs) to classify a total of 225 Raman spectra of natural and contaminated FM samples. Figure 3A shows the prediction results of cross validation (CV) based on individual spectra for different FM samples. A value of 1 corresponds to natural FM, a value of 2 corresponds to aspirin tabletcontaminated FM, a value of 3 corresponds to diclofenac tablet-contaminated FM, a value of 4 corresponds to ibuprofen tablet-contaminated FM, a value of 5 corresponds to ketoprofen tablet-contaminated FM and a value of 6 corresponds to naproxen tablet-contaminated FM, whereas a score of 0 indicated unassigned prediction. The CV prediction plot showed that 18 spectra were unassigned, and 4 spectra were misclassified, resulting in $90 \%$ correct classification of the cross validation test. Table S1 summarizes the prediction results on the PLS-DA model obtained from the CV test.

To improve the prediction results of the PLS-DA model, GA was proposed to select the regions that would be most informative for differentiating between the Raman spectra of different FM classes. GA is a machine learning technique that ultimately aims to optimize a given response function and is based on mimicking the theory of natural biological evolution. GA is a very useful method for variable selection in calibration and classification modeling, and more details about it can be found in the following article ${ }^{46}$. In this study, GA was carried out, and several spectral regions that noticeably contributed to the distinction between FM samples were selected, as 


\begin{tabular}{|c|c|c|c|}
\hline Raman band $\left(\mathrm{cm}^{-1}\right)$ & Source & Band assignment & Ref \\
\hline 1655 & Eccrine & $\mathrm{C}=\mathrm{O}$ stretching (secondary amide) & 31 \\
\hline $1629^{*}$ & Naproxen & Ring stretching, & 32 \\
\hline $1606^{*}$ & Aspirin & Ring stretching and $\mathrm{OH}$ bending & 33 \\
\hline $1606^{*}$ & Ibuprofen & Ring stretching & 32 \\
\hline $1606^{*}$ and 1578 & Diclofenac & Ring stretching & 37 \\
\hline $1598^{*}$ & Ketoprofen & $\mathrm{C}-\mathrm{C}$ stretching (ring) & 34 \\
\hline $1485^{\star}, 1420^{*}$ and 1168 & Naproxen & $\mathrm{CH}$ bending & 32 \\
\hline 1439 & Sebaceous & $\mathrm{CH}_{2}$ and $\mathrm{CH}_{3}$ deformation (aliphatic carbon chain) & 31 \\
\hline 1307 & Sebaceous & $\mathrm{CH}_{2}$ twisting (aliphatic carbon chain) & 31 \\
\hline 1267 & Sebaceous & $=\mathrm{CH}$ deformation (Squalene, unsaturated fatty acid, glycerides and wax esters & 31 \\
\hline 1250 & Diclofenac & $\mathrm{C}-\mathrm{C}$ stretching $\mathrm{CH}$ rock, & 35 \\
\hline 1236 & Diclofenac & $\mathrm{C}-\mathrm{N}-\mathrm{C}$ stretching, $\mathrm{CH}$ rock, $\mathrm{C}_{7} \mathrm{H}_{2}$ wagging & 35 \\
\hline $1207^{\star}$ and 1180 & Ibuprofen & $\mathrm{CH}$ bending and $\mathrm{OH}$ bending, & 32 \\
\hline $1194^{*}$ & Ketoprofen & Ring deformation and $\mathrm{C}-\mathrm{C}$ stretching & 34 \\
\hline $1194^{*}$ & Aspirin & $\Phi$, COC stretching ( $\Phi$ : ring) & 33 \\
\hline $1178^{*}$ & Naproxen & HCC in plane bending & 36 \\
\hline $1159^{*}$ & Aspirin & $\mathrm{CH}$ and $\mathrm{OH}$ in-plane bending & 32 \\
\hline $1159^{*}$ & Diclofenac & $\mathrm{CH}$ bending (ring) & 37 \\
\hline $1138^{*}$ & Ketoprofen & $\Phi$-C- $\Phi$ symmetric stretch ( $\Phi$ : ring) & 34 \\
\hline 1124 and 1078 & Sebaceous & C-C stretching (aliphatic carbon chain) & 31 \\
\hline 1116 & Ibuprofen & $\mathrm{CH}$ bending and $\mathrm{OH}$ bending, & 32 \\
\hline $1073^{\star}$ and $1045^{\star}$ & Diclofenac & Ring breathing & 37 \\
\hline $1045^{*}$ & Aspirin & $\mathrm{C}-\mathrm{H}$ bending & 33 \\
\hline $1031^{*}$ & Ketoprofen & $\mathrm{CH}$ in-plane bending & 34 \\
\hline $1009^{*}$ & Ibuprofen & $\mathrm{CH}$ in-plane bending & 32 \\
\hline $1002^{*}$ & Ketoprofen & Ring deformation and $\mathrm{CH}_{3}$ rocking & 34 \\
\hline 1002 & Eccrine & Ring breathing (phenyl alanine) & 31 \\
\hline 961 & Naproxen & Torsion-HCCH & 36 \\
\hline $861^{*}$ & Diclofenac & $\mathrm{CH}$ twisting & 37 \\
\hline $860^{*}$ & Eccrine & Para-substituted ring vibration (Tyrosine) & 31 \\
\hline $748^{*}$ & Naproxen & Torsion-HCCC & 36 \\
\hline $720^{*}$ & Diclofenac & $\mathrm{CH}$ wagging & 35 \\
\hline $704^{*}$ & Ketoprofen & $\mathrm{CH}$ out-of-plane bending & 34 \\
\hline 524 & Naproxen & HCC in-plane bending, Torsion-HCOC, Torsion-HCCO & 36 \\
\hline
\end{tabular}

Table 1. Raman band assignment for the natural and NSAID-contaminated fingermarks. The asterisks $\left(^{*}\right)$ indicate regions selected using the GA method.

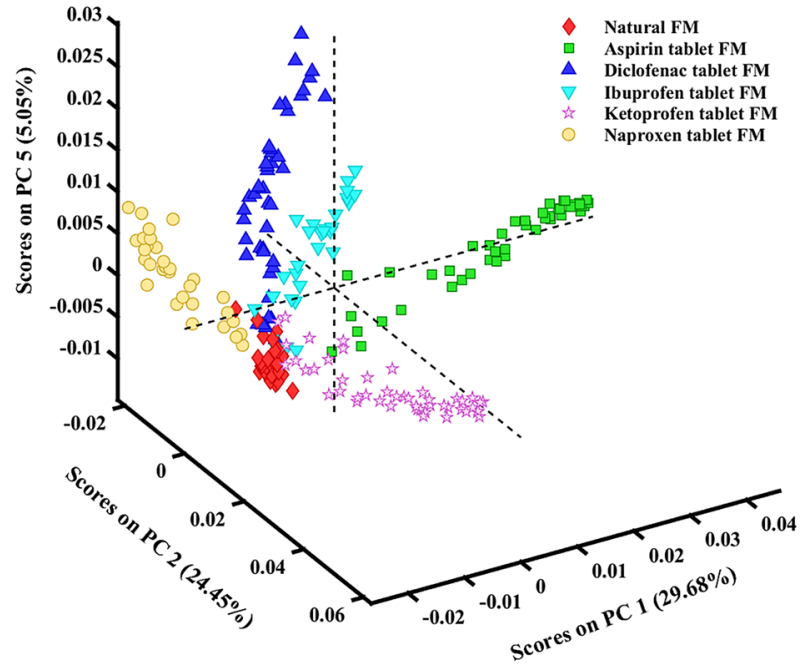

Figure 2. PCA scatter plot for individual Raman spectra of natural and NSAID-contaminated fingermarks. 
A

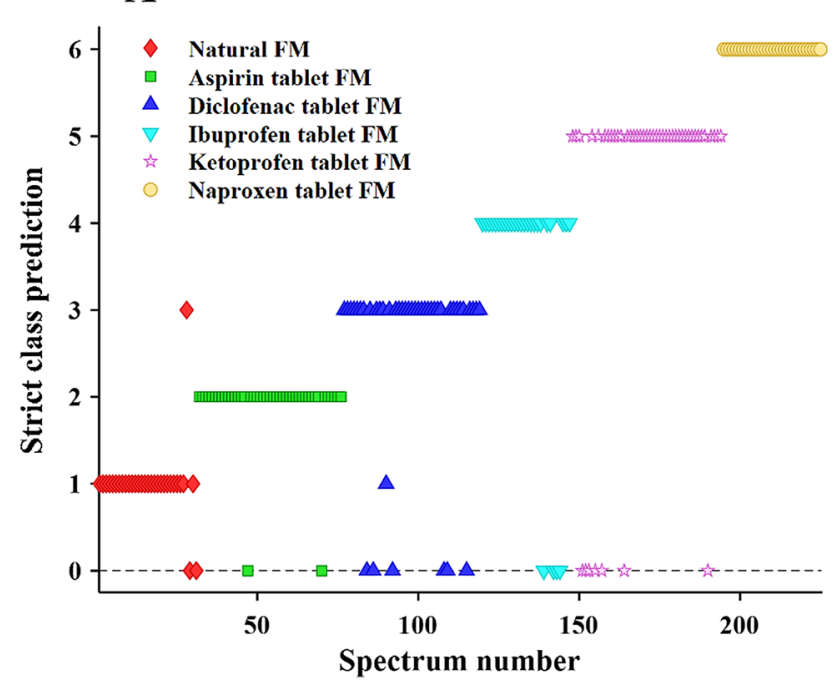

B

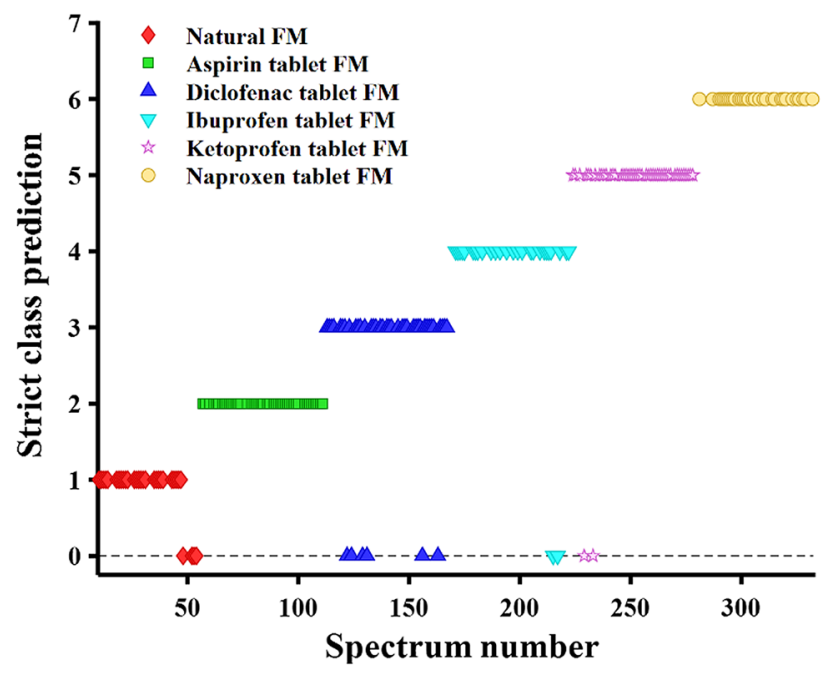

Figure 3. Cross validation prediction results of the PLS-DA model of each classification group using five latent variables: natural fingermark (red) and aspirin (green), diclofenac (navy blue), ibuprofen (light blue), ketoprofen (pink), and naproxen (orange) contaminated fingermarks (A) before applying GA and (B) after using the regions selected by GA.

illustrated in Table 1. Within the selected regions, the main difference can be attributed to ring stretching of the NSAID tablets at $1598-1629 \mathrm{~cm}^{-1}$. Regions were also selected at $1159-1207 \mathrm{~cm}^{-1}$ and assigned to the $-\mathrm{CH}$ and -OH bending of aspirin, diclofenac, ibuprofen and naproxen tablets, and at the peak at $1194 \mathrm{~cm}^{-1}$ was assigned to ring deformation $\mathrm{C}-\mathrm{C}$ stretching of the ketoprofen tablet. Other informative bands in the $1073-1002 \mathrm{~cm}^{-1}$ and $704-861 \mathrm{~cm}^{-1}$ regions were distinctive of NSAID tablets, suggesting their significant contribution to distinguishing between the Raman spectra of different FM classes. Figure 3B demonstrates the CV prediction results of the PLS-DA model using regions selected by GA. The results were drastically improved upon applying GA, yielding 94\% accuracy. Spectra of both the aspirin- and naproxen-contaminated FMs were perfectly assigned to their designated groups, while four spectra of natural FM and six spectra of diclofenac-contaminated FM were unassigned. In addition, both ketoprofen- and ibuprofen-contaminated FMs showed good separation in the model, and only two spectra were unassigned, while no misclassification was observed in the created model. This information is summarized in Table S2, which shows the confusion matrix of CV of the PLS-DA model using the strict class prediction method.

Method validation. To further support the reliability of the created model, external validation was performed using FMs provided by a new donor. Both natural and contaminated FMs were obtained from this donor, a total of 137 spectra were loaded into the model as unknown, and each spectrum was then assigned to a specific class. Figure 4 illustrates the prediction results of the external validation, specifically, 130 spectra were correctly assigned to the respective natural and contaminated FM classes, while only seven spectra were unassigned. This means that $94 \%$ of the total external validation spectra were correctly assigned to their corresponding FM classes, indicating the excellent performance of the constructed model, as outlined in Table 2. Most importantly, over $85 \%$ of all spectra obtained for an individual FM were assigned correctly for each of the six classes of FMs. This result indicates that if we choose a threshold of $85 \%$, then all samples used for the external validation are assigned correctly, demonstrating 100\% accuracy of the developed classification model. This proof-of-concept study offers a new approach to identifying natural and drug-contaminated FM components using Raman spectroscopy and multivariate statistical analysis.

\section{Conclusion}

This proof-of-concept study further develops Raman spectroscopy and chemometrics as a nondestructive and rapid method for the detection and identification of drugs in latent fingermarks (LFMs). Raman spectra were collected from natural FM and aspirin-, diclofenac-, ibuprofen-, ketoprofen- and naproxen-contaminated FMs obtained after the donor gently handled these tablets. Initially, PCA model was applied to identify spectral outliers, which were removed from the dataset before any further statistical analysis. Thereafter, multivariate PLS-DA and GA were employed to differentiate between natural and NSAID-contaminated FMs. The PLS-DA model was created using a training dataset and enabled an excellent separation of natural FM, aspirin-, diclofenac-, ibuprofen-, ketoprofen- and naproxen-contaminated FMs according to Venetian blind cross validation (CV). In addition, the method was externally validated using FM samples obtained from a second donor, and the results of strict class prediction showed $94 \%$ correct classification based on individual spectra. Most importantly, the individual samples showed $100 \%$ correct identification. Thus, the reported results demonstrate the great potential of Raman spectroscopy and chemometrics for the detection and identification of trace NSAIDs and potentially other drugs in LFMs. When fully developed and implemented in practical forensics, this methodology holds 


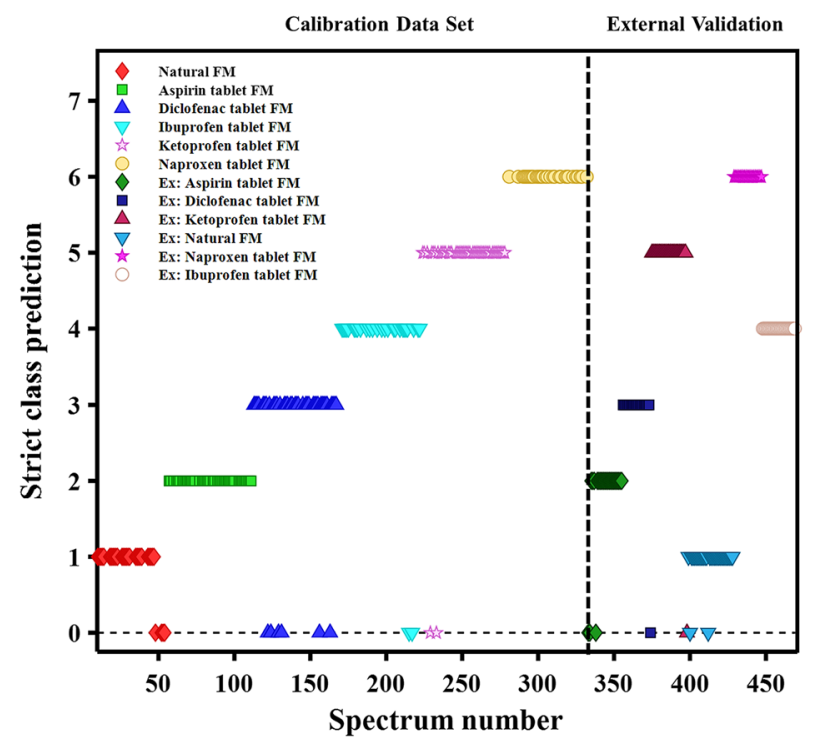

Figure 4. Strict prediction results of the PLS-DA classification model for natural FM- and NSAIDcontaminated FMs based on five latent variables using regions selected by GA. The left side of the plot shows the calibration results based on the training dataset. The right side of the plot shows the external validation tests from an independent donor.

\begin{tabular}{|c|c|c|c|c|c|c|}
\hline \multirow[b]{2}{*}{ Class predicted } & \multicolumn{6}{|l|}{ Actual class } \\
\hline & Natural FM & Aspirin tablet FM & $\begin{array}{l}\text { Diclofenac tablet } \\
\text { FM }\end{array}$ & $\begin{array}{l}\text { Ibuprofen tablet } \\
\text { FM }\end{array}$ & $\begin{array}{l}\text { Ketoprofen tablet } \\
\text { FM }\end{array}$ & $\begin{array}{l}\text { Naproxen tablet } \\
\text { FM }\end{array}$ \\
\hline Natural FM & 28 & 0 & 0 & 0 & 0 & 0 \\
\hline Aspirin tablet FM & 0 & 20 & 0 & 0 & 0 & 0 \\
\hline $\begin{array}{l}\text { Diclofenac tablet } \\
\text { FM }\end{array}$ & 0 & 0 & 18 & 0 & 0 & 0 \\
\hline $\begin{array}{l}\text { Ibuprofen tablet } \\
\text { FM }\end{array}$ & 0 & 0 & 0 & 22 & 0 & 0 \\
\hline $\begin{array}{l}\text { Ketoprofen tablet } \\
\text { FM }\end{array}$ & 0 & 0 & 0 & 0 & 23 & 0 \\
\hline $\begin{array}{l}\text { Naproxen tablet } \\
\text { FM }\end{array}$ & 0 & 0 & 0 & 0 & 0 & 19 \\
\hline Unclassified & 2 & 3 & 1 & 0 & 1 & 0 \\
\hline
\end{tabular}

Table 2. A confusion matrix for individual spectra obtained for the external validation of the PLS-DA model based on five latent variables using the regions selected by the GA method.

great promise in criminal investigations of drug overdose and handling, adulterant and suicide. Before the developed method can be applied by law enforcement agencies, further work is required to cover a wider range of drugs. In particular, we plan to expand the use of the current method and include several other drugs such as procuring and counterfeit drugs in addition to emerging synthetic cannabinoids in the future. Furthermore, the application of this method for examining other potential exogenous materials including explosives, gunshot residue, cosmetics and others in fingermarks will be also considered. Additionally, the variation in the chemical composition of the prints due to ungroomed or groomed types, potential interferences from environmental contaminants and common substrates as well as environmental conditions, including temperature, humidity, sunlight, should be addressed to simulate samples from real crime scenes.

Received: 6 October 2021; Accepted: 9 February 2022

Published online: 24 February 2022

\section{References}

1. Champod, C., Lennard, C. J., Margot, P., \& Stoilovic, M. Fingerprints and other ridge skin impressions. CRC press: 2004.

2. Hemmila, A., McGill, J. \& Ritter, D. Fourier transform infrared reflectance spectra of latent fingerprints: a biometric gauge for the age of an individual. J. Forensic Sci. 53(2), 369-376 (2008).

3. Ferguson, L. S. et al. Direct detection of peptides and small proteins in fingermarks and determination of sex by MALDI mass spectrometry profiling. Analyst 137(20), 4686-4692 (2012). 
4. Raiszadeh, M. M. et al. Proteomic analysis of eccrine sweat: Implications for the discovery of schizophrenia biomarker proteins. J. Proteome Res. 11(4), 2127-2139 (2012).

5. Francese, S. et al. Beyond the ridge pattern: Multi-informative analysis of latent fingermarks by MALDI mass spectrometry. Analyst 138(15), 4215-4228 (2013).

6. Bradshaw, R. et al. Spectroscopic imaging based approach for condom identification in condom contaminated fingermarks. Analyst 138(9), 2546-2557 (2013).

7. Weyermann, C., Roux, C. \& Champod, C. Initial results on the composition of fingerprints and its evolution as a function of time by GC/MS analysis. J. Forens. Sci. 56(1), 102-108 (2011).

8. Van Helmond, W. et al. Amino acid profiling from fingerprints, a novel methodology using UPLC-MS. Anal. Methods 9(38), 5697-5702 (2017).

9. Amin, M. O., Madkour, M. \& Al-Hetlani, E. Metal oxide nanoparticles for latent fingerprint visualization and analysis of small drug molecules using surface-assisted laser desorption/ionization mass spectrometry. Anal. Bioanal. Chem. 410(20), 4815-4827 (2018).

10. Muro, C. K., Doty, K. C., Bueno, J., Halamkova, L. \& Lednev, I. K. Vibrational spectroscopy: Recent developments to revolutionize forensic science. Anal Chem 87(1), 306-327 (2015).

11. Doty, K. C., Muro, C. K., Bueno, J., Halámková, L. \& Lednev, I. K. What can Raman spectroscopy do for criminalistics?. J. Raman Spectrosc. 47(1), 39-50 (2016).

12. Khandasammy, S. R. et al. Bloodstains, paintings, and drugs: Raman spectroscopy applications in forensic science. Forens. Chem 8, 111-133 (2018).

13. Amin, M. O., Al-Hetlani, E. \& Lednev, I. K. Trends in Vibrational Spectroscopy of Fingermarks for Forensic Purposes. TrAC, Trends Anal. Chem. 2021, 116341 (2021).

14. Ewing, A. V. \& Kazarian, S. G. Infrared spectroscopy and spectroscopic imaging in forensic science. Analyst 142(2), 257-272 (2017).

15. Ricci, C. et al. Chemical imaging of latent fingerprint residues. Appl. Spectrosc. 61(5), 514-522 (2007).

16. Ricci, C., Chan, K. A. \& Kazarian, S. G. In Fourier transform infared spectroscopic imaging for the identification of concealed drug residue particles and fingerprints 64020M (International Society for Optics and Photonics, 2006).

17. Ricci, C., Chan, K. A. \& Kazarian, S. G. Combining the tape-lift method and Fourier transform infrared spectroscopic imaging for forensic applications. Appl. Spectrosc. 60(9), 1013-1021 (2006).

18. Ricci, C., Bleay, S. \& Kazarian, S. G. Spectroscopic Imaging of Latent Fingermarks Collected with the Aid of a Gelatin Tape. Anal. Chem. 79(15), 5771-5776 (2007).

19. Ricci, C. \& Kazarian, S. G. Collection and detection of latent fingermarks contaminated with cosmetics on nonporous and porous surfaces. Surface Interface Anal. International Journal devoted to the development and application of techniques for the analysis of surfaces, interfaces and thin films 42(5), 386-392 (2010).

20. Almaviva, S. et al. A new eye-safe UV Raman spectrometer for the remote detection of energetic materials in fingerprint concentrations: Characterization by PCA and ROC analyzes. Talanta 144, 420-426 (2015).

21. Malka, I., Petrushansky, A., Rosenwaks, S. \& Bar, I. Detection of explosives and latent fingerprint residues utilizing laser pointerbased Raman spectroscopy. Appl. Phys. B 113(4), 511-518 (2013).

22. Day, J. S., Edwards, H. G., Dobrowski, S. A. \& Voice, A. M. The detection of drugs of abuse in fingerprints using Raman spectroscopy I: latent fingerprints. Spectrochim. Acta Part A Mol. Biomol. Spectrosc. 60(3), 563-568 (2004).

23. Day, J. S., Edwards, H. G., Dobrowski, S. A. \& Voice, A. M. The detection of drugs of abuse in fingerprints using Raman spectroscopy II: cyanoacrylate-fumed fingerprints. Spectrochim. Acta Part A Mol. Biomol. Spectrosc. 60(8-9), 1725-1730 (2004).

24. West, M. J. \& Went, M. J. The spectroscopic detection of drugs of abuse in fingerprints after development with powders and recovery with adhesive lifters. Spectrochim. Acta Part A Mol. Biomol. Spectrosc. 71(5), 1984-1988 (2009).

25. West, M. J. \& Went, M. J. The spectroscopic detection of exogenous material in fingerprints after development with powders and recovery with adhesive lifters. Forensic Sci. Int. 174(1), 1-5 (2008).

26. Widjaja, E. Latent fingerprints analysis using tape-lift, Raman microscopy, and multivariate data analysis methods. Analyst 134(4), $769-775$ (2009).

27. Wise, B., Gallagher, N., Bro, R., Shaver, J., Windig, W., \& Koch, R. S. PLS Toolbox 3.5 for use with Matlab. Manson, WA: Eigenvector Research Inc (2005).

28. Maurer, H. H., Tauvel, F. X. \& Kraemer, T. Screening procedure for detection of non-steroidal anti-inflammatory drugs and their metabolites in urine as part of a systematic toxicological analysis procedure for acidic drugs and poisons by gas chromatographymass spectrometry after extractive methylation. J. Anal. Toxicol. 25(4), 237-244 (2001).

29. Al-Hetlani, E., Halámková, L., Amin, M. O. \& Lednev, I. K. Differentiating smokers and nonsmokers based on Raman spectroscopy of oral fluid and advanced statistics for forensic applications. J. Biophoton. 13(3), 123 (2020).

30. Cui, L., Butler, H. J., Martin-Hirsch, P. L. \& Martin, F. L. Aluminium foil as a potential substrate for ATR-FTIR, transflection FTIR or Raman spectrochemical analysis of biological specimens. Anal. Methods 8(3), 481-487 (2016).

31. Dorakumbura, B. N. et al. Revealing the spatial distribution of chemical species within latent fingermarks using vibrational spectroscopy. Analyst 143(17), 4027-4039 (2018).

32. Mojica, E.-R. E., Zapata, J., Vedad, J., Desamero, R. Z., \& Dai, Z., Analysis of Over-the-Counter Drugs Using Raman Spectroscopy. In Raman Spectroscopy in the Undergraduate Curriculum, ACS Publications: 2018; pp 69-91.

33. Adomavičiūtè, S., Velička, M. \& Šablinskas, V. Detection of aspirin traces in blood by means of surface-enhanced Raman scattering spectroscopy. J. Raman Spectrosc. 51(6), 919-931 (2020).

34. Vueba, M., Pina, M., Veiga, F., Sousa, J. \& De Carvalho, L. B. Conformational study of ketoprofen by combined DFT calculations and Raman spectroscopy. Int. J. Pharm. 307(1), 56-65 (2006).

35. Iliescu, T., Baia, M. \& Kiefer, W. FT-Raman, surface-enhanced Raman spectroscopy and theoretical investigations of diclofenac sodium. Chem. Phys. 298(1-3), 167-174 (2004).

36. Saji, R. S. et al. Spectroscopic and quantum computational study on naproxen sodium. Spectrochimica Acta Part A Mol. Biomol. Spectrosc. 226, 117614 (2020).

37. Iliescu, T., Baia, M. \& Miclăuş, V. A Raman spectroscopic study of the diclofenac sodium- $\beta$-cyclodextrin interaction. Eur. J. Pharm. Sci. 22(5), 487-495 (2004).

38. Lee, L. C., Liong, C.-Y. \& Jemain, A. A. Partial least squares-discriminant analysis (PLS-DA) for classification of high-dimensional (HD) data: A review of contemporary practice strategies and knowledge gaps. Analyst 143(15), 3526-3539 (2018).

39. Lenhardt, L., Bro, R., Zeković, I., Dramićanin, T. \& Dramićanin, M. D. Fluorescence spectroscopy coupled with PARAFAC and PLS DA for characterization and classification of honey. Food Chem. 175, 284-291 (2015).

40. Ralbovsky, N. M. \& Lednev, I. K. Analysis of individual red blood cells for Celiac disease diagnosis. Talanta 221, 121642 (2021).

41. Arora, T. et al. Chemometrics based ATR-FTIR spectroscopy method for rapid and non-destructive discrimination between eyeliner and mascara traces. Microchem. J. 164, 1080 (2021).

42. Muro, C. K., Doty, K. C., Fernandes, L. \& d. S., Lednev, I. K.,. Forensic body fluid identification and differentiation by Raman spectroscopy. Forensic Chem 1(1), 31-38 (2016).

43. Mistek-Morabito, E. \& Lednev, I. K. Discrimination between human and animal blood by attenuated total reflection Fourier transform-infrared spectroscopy. Commun. Chem. 3(1), 1-6 (2020). 
44. Mistek-Morabito, E. \& Lednev, I. K. Discrimination of menstrual and peripheral blood traces using attenuated total reflection Fourier transform-infrared (ATR FT-IR) spectroscopy and chemometrics for forensic purposes. Anal. Bioanal. Chem. 413(9), 2513-2522 (2021)

45. Mistek, E., Halamkova, L. \& Lednev Igor, K. Phenotype profiling for forensic purposes: Nondestructive potentially on scene attenuated total reflection Fourier transform-infrared (ATR FT-IR) spectroscopy of bloodstains. Forensic Chem 216, 100176 (2019).

46. Niazi, A. \& Leardi, R. Genetic algorithms in chemometrics. J. Chemom. 26(6), 345-351 (2012).

\section{Acknowledgements}

The authors gratefully acknowledge Kuwait Foundation for the advancement of Sciences (KFAS) and the Department of Chemistry, Kuwait University for performing the required Raman analysis. E.H. and M.O.A. gratefully acknowledge support from the Kuwait University Research Administration (KURA), and the College of Graduate Studies (CGS) of Kuwait University. I.K.L. acknowledges support from the National Institute of Justice, Office of Justice Programs, U.S. Department of Justice.

\section{Author contributions}

M.O.A. performed the experiments, treated and analyzed spectral data, and drafted the manuscript. E.H. and I.K.L. conceived the project and were responsible for project administration and supervision, reviewing and editing the manuscript.

\section{Funding}

This work did not receive financial support from any funding agency.

\section{Competing interests}

The authors declare no competing interests.

\section{Additional information}

Supplementary Information The online version contains supplementary material available at https://doi.org/ 10.1038/s41598-022-07168-6.

Correspondence and requests for materials should be addressed to E.A.-H. or I.K.L.

Reprints and permissions information is available at www.nature.com/reprints.

Publisher's note Springer Nature remains neutral with regard to jurisdictional claims in published maps and institutional affiliations.

Open Access This article is licensed under a Creative Commons Attribution 4.0 International License, which permits use, sharing, adaptation, distribution and reproduction in any medium or format, as long as you give appropriate credit to the original author(s) and the source, provide a link to the Creative Commons licence, and indicate if changes were made. The images or other third party material in this article are included in the article's Creative Commons licence, unless indicated otherwise in a credit line to the material. If material is not included in the article's Creative Commons licence and your intended use is not permitted by statutory regulation or exceeds the permitted use, you will need to obtain permission directly from the copyright holder. To view a copy of this licence, visit http://creativecommons.org/licenses/by/4.0/.

(c) The Author(s) 2022 